PROCEEDINGS OF THE

AMERICAN MATHEMATICAL SOCIETY

Volume 137, Number 8, August 2009, Pages 2761-2765

S 0002-9939(09)09885-2

Article electronically published on March 18, 2009

\title{
OPTIMAL LENGTH ESTIMATES FOR STABLE CMC SURFACES IN 3-SPACE FORMS
}

\author{
LAURENT MAZET
}

(Communicated by Richard A. Wentworth)

\begin{abstract}
In this paper, we study stable constant mean curvature $H$ surfaces in $\mathbb{R}^{3}$. We prove that, in such a surface, the distance from a point to the boundary is less than or equal to $\pi /(2 H)$. This upper bound is optimal and is extended to stable constant mean curvature surfaces in space forms.
\end{abstract}

\section{INTRODUCTION}

A constant mean curvature (cmc) surface $\Sigma$ in a Riemannian 3-manifold $\mathbb{M}^{3}$ is stable if its stability operator, $L=-\Delta-\operatorname{Ric}(n, n)-|A|^{2}$, is nonnegative, where $\Delta$ is the Laplace operator on $\Sigma$, Ric is the Ricci tensor on $\mathbb{M}^{3}, n$ is the normal along $\Sigma$ and $A$ is the second fundamental form on $\Sigma$. For minimal surfaces $(H=0)$, this characterization is only valid for two-sided surfaces, so in the following we restrict ourselves to such surfaces. The nonnegativity of the stability operator means that $\Sigma$ is a local minimizer of the area functional on surfaces with regard to the infinitesimal deformations fixing its boundary.

The stability hypothesis was studied by several authors and has many consequences (see [6] for an overview). For example, D. Fischer-Colbrie and R. Schoen [4] studied the case of complete stable minimal surfaces when $\mathbb{M}^{3}$ has nonnegative scalar curvature. They obtain that the universal cover of $\Sigma$ is not conformally equivalent to the disk and, as a consequence, prove that the plane is the only complete stable minimal surface in $\mathbb{R}^{3}$. From this, R. Schoen [9] has derived a curvature estimate for stable cmc surfaces.

In 2, T. H. Colding and W. P. Minicozzi introduced new techniques and obtained area and curvature estimates for stable cmc surfaces. Afterward, these techniques were used by P. Castillon 1 to answer a question asked in 4 about the consequences of the positivity of certain elliptic operators. Recently, the same ideas have been used by J. Espinar and H. Rosenberg 3 to obtain similar results.

In [7, A. Ros and H. Rosenberg study constant mean curvature $H$ surfaces in $\mathbb{R}^{3}$ with $H \neq 0$. They prove a maximum principle at infinity. One of their tools is a length estimate for stable cmc surfaces. In fact, they prove that the intrinsic distance from a point $p$ in a stable cmc surface $\Sigma$ to the boundary of $\Sigma$ is less than $\pi / H$. H. Rosenberg [8] has generalized this result to any ambient 3-manifolds and large mean curvature. The aim of this paper is to improve the result of Ros

Received by the editors September 26, 2008, and, in revised form, January 7, 2009.

2000 Mathematics Subject Classification. Primary 53A10.

(C)2009 American Mathematical Society 
and Rosenberg. In fact, applying the ideas of [2], we prove that the distance is less than $\pi /(2 H)$. This estimate is optimal since, for a hemisphere of radius $1 / H$, the distance from the pole to the boundary is $\pi /(2 H)$. Actually we prove that the hemisphere of radius $1 / H$ is the only stable cmc $H$ surface where the distance $\pi /(2 H)$ is reached. We can generalize this result to stable $\mathrm{cmc} H$ surfaces in $\mathbb{M}^{3}(\kappa)$, where $\mathbb{M}^{3}(\kappa)$ is the 3-space form of sectional curvature $\kappa$. We prove that when $H^{2}+\kappa>0$ such an optimal estimate exists. In fact, it is already known that when $\kappa \leq 0$ and $H^{2}+\kappa \leq 0$, there is no such estimate since there exist complete stable cmc $H$ surfaces. But, in some sense, our results are an extension of the fact that the planes (resp. the horospheres) are the only stable complete constant mean curvature $H$ surfaces in $\mathbb{R}^{3}$ (resp. $\left.M^{3}(\kappa), \kappa<0\right)$ when $H=0\left(\right.$ resp. $\left.H^{2}+\kappa=0\right)$.

\section{Definitions}

On a constant mean curvature surface $\Sigma$ in a Riemannian 3-manifold $\mathbb{M}^{3}$, the stability operator is defined by $L=-\Delta-\operatorname{Ric}(n, n)-|A|^{2}$, where $\Delta$ is the Laplace operator on $\Sigma$, Ric is the Ricci tensor on $\mathbb{M}^{3}, n$ is the normal along $\Sigma$ and $A$ is the second fundamental form on $\Sigma$. When it is necessary, we will denote the stability operator by $L_{f}$ to refer to the immersion $f$ of $\Sigma$ in $\mathbb{M}^{3}$.

The surface $\Sigma$ is called stable if the operator $L$ is nonnegative; i.e., for every compactly supported function $u$, we have

$$
0 \leq \int_{\Sigma} u L(u) \mathrm{d} \sigma=\int_{\Sigma}\|\nabla u\|^{2}-\left(\operatorname{Ric}(n, n)+|A|^{2}\right) u^{2} \mathrm{~d} \sigma .
$$

We remark that this property is sometimes called strong stability since it means that the second derivatives of the area functional are nonnegative with respect to any compactly supported infinitesimal deformations $u$, whereas $\Sigma$ is critical for this functional only for compactly supported infinitesimal deformations with vanishing mean value, i.e. $\int_{\Sigma} u \mathrm{~d} \sigma=0$.

In the following, on a cmc surface, the normal $n$ is always chosen such that $H$ is nonnegative.

We will denote by $d_{\Sigma}$ the intrinsic distance on $\Sigma$ and by $K$ the sectional curvature of the surface.

\section{Results}

The main result of this paper is the following theorem.

Theorem 3.1. Let $H$ be positive. Let $\Sigma$ be a stable constant mean curvature $H$ surface in $\mathbb{R}^{3}$. Then, for $p \in \Sigma$, we have

$$
d_{\Sigma}(p, \partial \Sigma) \leq \frac{\pi}{2 H}
$$

Moreover, if the equality is satisfied, $\Sigma$ is a hemisphere.

In $\mathbb{R}^{3}$, the stability operator can be written $L=-\Delta-4 H^{2}+2 K$.

Proof. We denote by $R_{0}$ the distance $d_{\Sigma}(p, \partial \Sigma)$ and assume that $R_{0} \geq \pi /(2 H)$. If $R_{0}<\pi / H$ we denote by $I$ the segment $\left[\pi /(2 H), R_{0}\right]$; otherwise $I=[\pi /(2 H), \pi / H)$. In fact, because of the work of Ros and Rosenberg [7, we already know that $R_{0} \leq$ $\pi / H$. Let $R$ be in $I$.

The surface $\Sigma$ has constant mean curvature $H$; thus its sectional curvature is less than $H^{2}$. So the exponential map $\exp _{p}$ is a local diffeomorphism on the disk 
$D(0, R) \subset T_{p} \Sigma$ of center 0 and radius $R$. On this disk, we consider the induced metric and the operator $\mathcal{L}=-\Delta-4 H^{2}+2 K$. The surface $\Sigma$ is stable, so there exists a positive function $g$ on $\Sigma$ such that $L(g)=0$ (see Theorem 1 in 4 ). On $D(0, R)$, the function $\tilde{g}=g \circ \exp _{p}$ is then positive and satisfies $\mathcal{L}(\tilde{g})=0$ since $D(0, R)$ and $\Sigma$ are locally isometric. The operator $\mathcal{L}$ is thus nonnegative on $D(0, R)$ 4].

For $r \in[0, R]$, we define $l(r)$ as the length of the circle $\{v,|v|=r\} \subset D(0, R)$ and $\mathcal{K}(r)=\int_{D(0, r)} K \mathrm{~d} \sigma$. Since $D(0, R)$ and $\Sigma$ are locally isometric, the sectional curvature $K$ of $D(0, R)$ is less than $H^{2}$. Then

$$
l(r) \geq \frac{2 \pi}{H} \sin H r .
$$

By Gauss-Bonnet, we have

$$
\mathcal{K}(r)=2 \pi-l^{\prime}(r)
$$

Let us consider a function $\eta:[0, R] \rightarrow[0,1]$ with $\eta(0)=1$ and $\eta(R)=0$. Let us write the nonnegativity of $\mathcal{L}$ for the radial function $u=\eta(r)$ :

$$
0 \leq \int_{0}^{R}\left(\eta^{\prime}(r)\right)^{2} l(r) \mathrm{d} r-4 H^{2} \int_{0}^{R} \eta^{2}(r) l(r) \mathrm{d} r+2 \int_{0}^{R} \mathcal{K}^{\prime}(r) \eta^{2}(r) \mathrm{d} r .
$$

Hence, following the ideas in 2 and using (3.3) and the boundary values of $\eta$, we have

$$
\begin{aligned}
\int_{0}^{R}\left(4 H^{2} \eta^{2}-{\eta^{\prime}}^{2}\right) l \mathrm{~d} r \leq 2 & \left(\left[\mathcal{K}(r) \eta^{2}(r)\right]_{0}^{R}-\int_{0}^{R} \mathcal{K}(r)\left(\eta^{2}(r)\right)^{\prime} \mathrm{d} r\right) \\
& =-2 \int_{0}^{R} \mathcal{K}(r)\left(\eta^{2}(r)\right)^{\prime} \mathrm{d} r \\
& =-2 \int_{0}^{R}\left(2 \pi-l^{\prime}(r)\right)\left(\eta^{2}(r)\right)^{\prime} \mathrm{d} r \\
& =4 \pi+2 \int_{0}^{R}\left(\eta^{2}(r)\right)^{\prime} l^{\prime}(r) \mathrm{d} r \\
& =4 \pi+\left[2\left(\eta^{2}(r)\right)^{\prime} l(r)\right]_{0}^{R}-2 \int_{0}^{R}\left(\eta^{2}(r)\right)^{\prime \prime} l(r) \mathrm{d} r \\
& =4 \pi-2 \int_{0}^{R}\left(\eta^{2}(r)\right)^{\prime \prime} l(r) \mathrm{d} r .
\end{aligned}
$$

Thus we obtain

$$
\int_{0}^{R}\left(4 H^{2} \eta^{2}-\eta^{\prime 2}+2\left(\eta^{2}\right)^{\prime \prime}\right) l \mathrm{~d} r \leq 4 \pi
$$

We shall apply this equation to the function $\eta(r)=\cos \frac{\pi r}{2 R}$. In this case we have

$$
\begin{aligned}
\eta^{2} & =\frac{\pi^{2}}{4 R^{2}} \sin ^{2} \frac{\pi r}{2 R}, \\
\left(\eta^{2}\right)^{\prime \prime} & =-\frac{\pi^{2}}{2 R^{2}}\left(\cos ^{2} \frac{\pi r}{2 R}-\sin ^{2} \frac{\pi r}{2 R}\right) .
\end{aligned}
$$

Thus

$$
4 H^{2} \eta^{2}-\eta^{\prime 2}+2\left(\eta^{2}\right)^{\prime \prime}=\left(4 H^{2}-\frac{\pi^{2}}{R^{2}}\right) \cos ^{2} \frac{\pi r}{2 R}+\frac{3 \pi^{2}}{4 R^{2}} \sin ^{2} \frac{\pi r}{2 R} .
$$


As $R \geq \frac{\pi}{2 H}, 4 H^{2} \eta^{2}-\eta^{\prime 2}+2\left(\eta^{2}\right)^{\prime \prime}$ is nonnegative and, by (3.2),

$$
\begin{gathered}
\left(4 H^{2} \eta^{2}-\eta^{\prime 2}+2\left(\eta^{2}\right)^{\prime \prime}\right) l \geq\left(\left(4 H^{2}-\frac{\pi^{2}}{R^{2}}\right) \cos ^{2} \frac{\pi r}{2 R}+\frac{3 \pi^{2}}{4 R^{2}} \sin ^{2} \frac{\pi r}{2 R}\right) \frac{2 \pi}{H} \sin H r \\
\geq \frac{\pi}{H}\left(\left(4 H^{2}-\frac{\pi^{2}}{4 R^{2}}\right) \sin H r+\left(4 H^{2}-\frac{7 \pi^{2}}{4 R^{2}}\right) \frac{1}{2}\left(\sin \left(\frac{\pi}{R}+H\right) r-\sin \left(\frac{\pi}{R}-H\right) r\right)\right)
\end{gathered}
$$

Thus integrating in (3.4), we obtain (we recall that $R<\pi / H$ )

$$
\begin{aligned}
4 \pi \geq \frac{\pi}{H}( & \left(4 H^{2}-\frac{\pi^{2}}{4 R^{2}}\right) \frac{1}{H}(1-\cos H R) \\
& \left.\quad+\left(4 H^{2}-\frac{7 \pi^{2}}{4 R^{2}}\right) \frac{1}{2}\left(\frac{R}{\pi+H R}(1-\cos (\pi+H R))-\frac{R}{\pi-H R}(1-\cos (\pi-H R))\right)\right) .
\end{aligned}
$$

After some simplifications in the above expression, we obtain

$$
4 \pi \geq \pi \frac{\left(-32 H^{2} R^{4}+24 \pi^{2} H^{2} R^{2}-\pi^{4}\right)-\left(10 \pi^{2} H^{2} R^{2}-\pi^{4}\right) \cos H R}{4 H^{2} R^{2}\left(\pi^{2}-H^{2} R^{2}\right)} .
$$

Now, passing $4 \pi$ on the right-hand side of the above inequality and simplifying by $\pi$, we get

$$
F(R):=\frac{-\left(4 H^{2} R^{2}-\pi^{2}\right)^{2}-\left(10 \pi^{2} H^{2} R^{2}-\pi^{4}\right) \cos H R}{4 H^{2} R^{2}\left(\pi^{2}-H^{2} R^{2}\right)} \leq 0 .
$$

If we write $R=\pi /(2 H)+x$, we compute the Taylor expansion of $F$ and obtain

$$
F\left(\frac{\pi}{2 H}+x\right)=2 H x+o(x),
$$

which is positive if $x>0$. Thus, if $R_{0}>\pi /(2 H)$, we get a contradiction and the inequality (3.1) is proved.

Now if $R_{0}=\pi /(2 H)$, we have in fact equality all along the computation, so $l(r)=(2 \pi / H) \sin H r$ and $\mathcal{K}(r)=2 \pi-l^{\prime}(r)=2 \pi(1-\cos H r)$. But we also know that the sectional curvature is less than $H^{2}$; thus $\mathcal{K}(r) \leq H^{2} \int_{0}^{r} l(u) \mathrm{d} u=2 \pi(1-\cos H r)$. Since this inequality is in fact an equality, the sectional curvature is in fact $H^{2}$ at every point. Thus the principal curvatures of a point in $\Sigma$ are $H$ and $H$; i.e. there are only umbilical points. Hence $\Sigma$ is a piece of a sphere of radius $1 / H$ and, since $d_{\Sigma}(p, \partial \Sigma)=\frac{\pi}{2 H}$, it contains the hemisphere of pole $p$. A hemisphere cannot be strictly contained in a stable subdomain of the sphere, so $\Sigma$ is a hemisphere.

With this result we have an important corollary.

Corollary 3.2. Let $H \geq 0$ and $\kappa \in \mathbb{R}$ such that $H^{2}+\kappa>0$. Let $\Sigma$ be a stable contant mean curvature $H$ surface in $\mathbb{M}^{3}(\kappa)$. Then for $p \in \Sigma$, we have

$$
d_{\Sigma}(p, \partial \Sigma) \leq \frac{\pi}{2 \sqrt{H^{2}+\kappa}}
$$

Moreover, if the equality is satisfied, $\Sigma$ is a geodesical hemisphere of $\mathbb{M}^{3}(\kappa)$.

The proof is based on the Lawson correspondence between constant mean curvature surfaces in space forms (see [5]).

Proof. First, the case $\kappa=0$ is Theorem 3.1 .

Let $\Pi: \widetilde{\Sigma} \rightarrow \Sigma$ be the universal cover of $\Sigma$. We then have a constant mean curvature immersion of $\widetilde{\Sigma}$ in $\mathbb{M}^{3}(\kappa)$. Let $\mathcal{L}=-\Delta-2 \kappa-|A|^{2}$ be the stability operator on $\widetilde{\Sigma}$. $\Sigma$ is stable, so there exists a positive function $g$ on $\Sigma$ such that $L(g)=-\Delta g-\left(2 \kappa+|A|^{2}\right) g=0$. Thus the function $\tilde{g}=g \circ \Pi$ is a positive function 
on $\widetilde{\Sigma}$ satisfying $\mathcal{L}(\tilde{g})=0$. Hence $\widetilde{\Sigma}$ is stable. Let I and $S$ be respectively the first fundamental form and the shape operator on $\widetilde{\Sigma}$. They satisfy the Gauss and Codazzi equations for $\mathbb{M}^{3}(\kappa)$.

We define $S^{\prime}=S+\left(-H+\sqrt{H^{2}+\kappa}\right)$ id on $\widetilde{\Sigma}$. Then I and $S^{\prime}$ satisfy the Gauss and Codazzi equations for $\mathbb{M}^{3}(0)=\mathbb{R}^{3}$ (see [5]). Hence there exists an immersion $f$ of $\widetilde{\Sigma}$ in $\mathbb{R}^{3}$ with first fundamental form I and shape operator $S^{\prime}$ (we notice that the induced metric is the same). Its mean curvature is then $H+\left(-H+\sqrt{H^{2}+\kappa}\right)=$ $\sqrt{H^{2}+\kappa} ;$ i.e. the immersion has constant mean curvature. The stability operator is

$$
\begin{aligned}
L_{f} & =-\Delta-\left\|S^{\prime}\right\|^{2} \\
& =-\Delta-\left(\|S\|^{2}+4 H\left(-H+\sqrt{H^{2}+\kappa}\right)+2\left(-H+\sqrt{H^{2}+\kappa}\right)^{2}\right) \\
& =-\Delta-\left(\|S\|^{2}+2 \kappa\right) \\
& =\mathcal{L} .
\end{aligned}
$$

Hence the surface $f(\widetilde{\Sigma})$ is stable. So, from Theorem 3.1, we have

where $\Pi(\tilde{p})=p$.

$$
d_{\Sigma}(p, \partial \Sigma)=d_{\widetilde{\Sigma}}(\tilde{p}, \partial \widetilde{\Sigma}) \leq \frac{\pi}{2 \sqrt{H^{2}+\kappa}}
$$

The equality case comes from the equality case in Theorem 3.1 and since the Lawson correspondence sends spheres into spheres.

\section{REFERENCES}

[1] Philippe Castillon. An inverse spectral problem on surfaces. Comment. Math. Helv., 81:271286, 2006. MR2225628(2007b:58042)

[2] Tobias H. Colding and William P. Minicozzi II. Estimates for parametric elliptic integrands. Int. Math. Res. Not., 2002(6):291-297. MR1877004 (2002k:53060)

[3] Jose M. Espinar and Harold Rosenberg. A Colding-Minicozzi stability inequality and its applications, to appear in Trans. Amer. Math. Soc.

[4] Doris Fischer-Colbrie and Richard Schoen. The structure of complete stable minimal surfaces in 3-manifolds of nonnegative scalar curvature. Comm. Pure Appl. Math., 33:199-211, 1980. MR.562550(81i:53044)

[5] H. Blaine Lawson, Jr. Complete minimal surfaces in $S^{3}$. Ann. of Math. (2), 92:335-374, 1970. MR0270280(42:5170)

[6] William H. Meeks III, Joaquín Pérez, and Antonio Ros. Stable constant mean curvature surfaces, preprint.

[7] Antonio Ros and Harold Rosenberg. Properly embedded surfaces with constant mean curvature, preprint.

[8] Harold Rosenberg. Constant mean curvature surfaces in homogeneously regular 3-manifolds. Bull. Austral. Math. Soc., 74:227-238, 2006. MR2260491(2007g:53009)

[9] Richard Schoen. Estimates for stable minimal surfaces in three-dimensional manifolds. In Seminar on minimal submanifolds, volume 103 of Ann. of Math. Stud., pages 111-126. Princeton Univ. Press, 1983. MR795231 (86j:53094)

Laboratoire D'Analyse et Mathématiques Appliquées, Université Paris-Est, CNRS UMR8050, UFR des Sciences et Technologie, BÂtiment P3 4eme Étage, 61 avenue du Général de Gaulle, 94010 Créteil Cedex, France

E-mail address: laurent.mazet@math.cnrs.fr 